\title{
Role of targeted and universal mupirocin-based decolonization for preventing surgical-site infections in patients undergoing cardiothoracic surgery: A systematic review and meta-analysis
}

\author{
LI WANG $^{1}$, QI JI ${ }^{1}$ and XIAOYAN HU ${ }^{2}$ \\ Departments of ${ }^{1}$ Operating Room and ${ }^{2}$ Tongguan Operating Room, \\ The First People's Hospital of Lianyungang City, Lianyungang, Jiangsu 222002, P.R. China
}

Received June 10, 2020; Accepted September 11, 2020

DOI: $10.3892 /$ etm.2021.9860

\begin{abstract}
The purpose of the present study was to provide a systematic literature review and pool evidence on the efficacy of mupirocin-based decolonization protocol in reducing surgical-site infections (SSIs) in patients undergoing cardiothoracic (CT) surgery based on their Staphylococcus (S.) aureus carrier state. The PubMed, Embase, Ovid, BioMed Central, Cochrane Central Register of Controlled Trials and Google Scholar databases were searched for studies comparing mupirocin-based decolonization with controls for reducing SSIs in patients following CT surgery. Studies were grouped based on the targeted population of intervention, i.e. carriers or all patients. A total of 17 studies were included. Of these, 8 studies used targeted mupirocin-based decolonization, while universal decolonization was performed in 9 studies. The results were conflicting for studies performing targeted decolonization and it was not possible to perform a meta-analysis due to non-homogenous studies. Pooled analysis of 34,859 patients indicated that universal mupirocin-based decolonization significantly reduced the risk of all SSIs [risk ratio (RR): 0.54; 95\% CI: $0.40,0.75 ; \mathrm{I}^{2}=73.35 \%$ ]. The intervention significantly reduced the risk of superficial SSIs (RR: $0.37 ; 95 \%$ CI: $0.25,0.55 ; \mathrm{I}^{2}=0 \%$ ) but not of deep SSIs (RR: 0.45 ; 95\% CI: $0.19,1.09 ; \mathrm{I}^{2}=80.67 \%$ ). The results indicated a significantly reduced risk of $S$. aureus SSIs (SA-SSIs) with mupirocin-based decolonization (RR: 0.44; $95 \% \mathrm{CI}$ : $0.32,0.61 ; \mathrm{I}^{2}=0 \%$ ) but not for methicillin-resistant $S$. aureus
\end{abstract}

Correspondence to: Mrs. Qi Ji, Department of Operating Room, The First People's Hospital of Lianyungang City, 8 Zhenhua East Road, Lianyungang, Jiangsu 222002, P.R. China

E-mail: jiqicat@163.com

Mrs. Xiaoyan Hu, Tongguan Operating Room, The First People's Hospital of Lianyungang City, 8 Zhenhua East Road, Lianyungang, Jiangsu 222002, P.R. China

E-mail: 15878020494@139.com

Key words: Staphylococcus aureus, nosocomial infection, nasal carriers, cardiac surgery, infection, antibiotic
(MRSA-SSIs; RR: 0.25; 95\% CI: 0.05,1.28; $\mathrm{I}^{2}=79.07 \%$ ). Evidence on the role of targeted mupirocin-based decolonization to reduce SSIs after CT surgery was non-coherent and inconclusive. Analysis of low-quality retrospective studies suggested that universal mupirocin-based decolonization may reduce all SSIs, superficial SSIs and SA-SSIs, but not deep SSIs or MRSA-SSIs in patients after CT surgery.

\section{Introduction}

Nursing personnel has an important role in the pre-operative and post-operative management of patients in any surgical practice. Enforcement of strict infection control measures by them may significantly influence the rates of nosocomial surgical site infections (SSIs) in a health-care setup.

SSIs are an important cause of increased morbidity and mortality after cardiothoracic (CT) surgery (1). According to the literature, the reported incidence of SSIs after CT surgery varies from 3 to $10.5 \%$ (1-3). A small proportion of these SSIs are able to progress to deep sternal or mediastinal wound infection, which requires aggressive medical and surgical therapy and the associated mortality rate is up to $47 \%(4,5)$. Approximately $50 \%$ of SSIs after CT surgery are caused by Staphylococcus aureus (S. aureus), while coagulase-negative staphylococci, gram-negative bacteria and yeast are less frequently implicated $(3,6)$.

$S$. aureus is known to colonize the skin and mucosae of $15-36 \%$ of the global population (7). Of the various sites reported, the nose is considered to be an important reservoir in humans and nasal colonization has been reported to be an independent risk factor for SSIs (7-9). To reduce the incidence of $S$. aureus infections, nasal application of topical mupirocin has been widely reported in the literature $(10,11)$. In one of the earliest randomized controlled trials (RCTs) on surgical patients, Bode et al (12) reported a significantly reduced incidence of SSIs in S. aureus carriers with the use of pre-operative nasal mupirocin and chlorhexidine soap. A Cochrane review by van Rijen et al (10), analyzing nine RCTs, indicated that nasal mupirocin was associated with a statistically significant reduction of $S$. aureus infection. The review, however, included studies irrespective of the site of the surgical procedure. The incidence of SSI may depend on several factors, including 
patient characteristics, duration of surgical procedure and factors specific to the operative site (3). While factors such as rheumatoid arthritis, psoriasis, immunosuppression or history of surgery at the site of the prosthesis may influence the risk of SSI in orthopedic surgery, infections after CT surgery may be impacted by the body mass index, hemodialysis, cardiogenic shock, perfusion time, use of intra-aortic pump and the presence of $>3$ anastomoses (3). It is important to further determine the risk of SSIs for specific surgical sites.

Evidence on the use of mupirocin-based decolonization specifically in CT surgery has been rather conflicting. Konvalinka et al (13) reported on an RCT that indicated no beneficial effect of prophylactic nasal mupirocin in reducing the incidence of SSI after CT surgery. On the other hand, in a recent study, Lemaignen et al (14) reported a significant reduction in the incidence of SSIs with the use of nasal mupirocin-based decolonization protocol in their 13-year experience. Despite several reviews and meta-analyses assessing the role of mupirocin-based decolonization in reducing $S$. aureus infection (10,15-17), only a small number of studies have analyzed evidence on the efficacy of such an intervention in reducing SSIs specifically in patients following CT surgery. A meta-analysis by Schweizer et al (17) from 2013 assessed the efficacy of bundled intervention consisting of nasal decolonization (not restricted to mupirocin) and glycopeptide prophylaxis to reduce SSIs in patients subjected to cardiac or orthopedic surgery. They reported a statistically significant reduction of SSIs with nasal decolonization in cardiac patients but did not separate cardiac studies based on the targeted population treated with mupirocin (i.e. S. aureus carriers or all patients). Similarly, Ma et al (18) performed a meta-analysis of pediatric and adult patients subjected to CT surgery, pooling data of studies utilizing both targeted and universal decolonization for reducing SSIs.

Considering the lacunae in the literature, the purpose of the present review was to perform a systematic literature search and pool evidence on the role of the mupirocin-based decolonization protocol in reducing SSIs in patients undergoing CT surgery based on their $S$. aureus carrier status.

\section{Materials and methods}

Search strategy. A computerized literature search of the PubMed (https://pubmed.ncbi.nlm.nih.gov), Embase (https://www.embase.com), Ovid (https://ovidsp.ovid. com), BioMed Central (https://www.biomedcentral.com), Cochrane Central Register of Controlled Trials (CENTRAL; https://www.cochranelibrary.com/central/about-central) and Google scholar (https://scholar.google.com) databases was performed. The search was conducted by two reviewers independently (QJ and XH). The same reviewers independently screened the databases from inception up to 1st March 2020. $\mathrm{MeSH}$ terms, as well as free-text keywords, were used in the literature search. The entire search protocol, as well as the results of the PubMed database, are presented in Table SI. The search results were screened by their titles and abstracts for each database. Potentially relevant articles were then extracted and subsequently screened by their full text. Both the reviewers assessed individual studies based on inclusion criteria and resolved any disagreement by discussion. After screening, the bibliography of included studies, as well as review articles on the subject, were hand searched for any additional references. The Preferred Reporting Items for Systematic Reviews and Meta-analyses guidelines were followed during the conduct of this systematic review, except for protocol registration (19).

Inclusion criteria. All types of studies were included in this systematic review. Included studies were to be performed on adult patients (age, $>18$ years) undergoing any type of CT surgery ('Participants'). The study intervention was nasal decolonization with mupirocin ointment in the study participants. Studies with bundled interventions utilizing mupirocin-based nasal decolonization were also included. Studies utilizing targeted decolonization of $S$. aureus carriers as well as those performing universal decolonization of all patients were included. Studies were to compare the study group with a control group for the incidence of SSIs. Studies not reporting relevant data or separate data for CT surgery patients and those not specifying the targeted population of mupirocin-based decolonization (carriers or all) were excluded. Single-arm studies, case series, case reports, abstracts, non-English language studies and review articles were also excluded.

Data extraction and quality of included studies. Using a pre-formatted abstraction form, the reviewers extracted data from the included studies (QJ and XH). Details including the name of the first author, publication year, study type, study location, sample size, demographic details, use of screening for carrier state and method of screening, decolonization protocol, antibiotic prophylaxis use and incidence of SSIs were extracted. The primary outcome variable was all SSIs irrespective of the causative organism and site. Secondary outcomes were superficial SSIs, deep SSIs, SSIs caused by S. aureus (SA-SSIs) and SSIs caused by MRSA (MRSA-SSIs). Definitions of SSIs were as per the included studies.

The risk of bias of RCTs was assessed using the Cochrane Collaboration risk assessment tool for RCTs (20). Each study was assessed in the following domains: Random sequence generation, allocation concealment, blinding of participants and personnel, blinding of outcome assessment, incomplete outcome data, selective reporting and other biases. The risk of a bias assessment tool for non-randomized studies was used to assess non-RCTs (21). Studies were rated as having low risk, high risk or unclear risk of bias in the following categories: Selection of participants, confounding variables, intervention measurements, blinding of outcome assessment, incomplete outcome data and selective outcome reporting.

Statistical analysis. Studies were divided into two groups based on the decolonization protocol. The first group consisted of studies utilizing decolonization of only carriers after appropriate pre-operative screening (targeted decolonization), while the second group consisted of studies carrying out decolonization of all patients in the study group irrespective of the carrier state (universal decolonization). Studies were pooled for a meta-analysis only if they were conducted on coherent groups and reported data on the same scale. The software 'Open MetaAnalyst (version Yosemite 10.10)' was used for the meta-analysis (22). To take methodological heterogeneity of the included studies into account, a random-effects model 

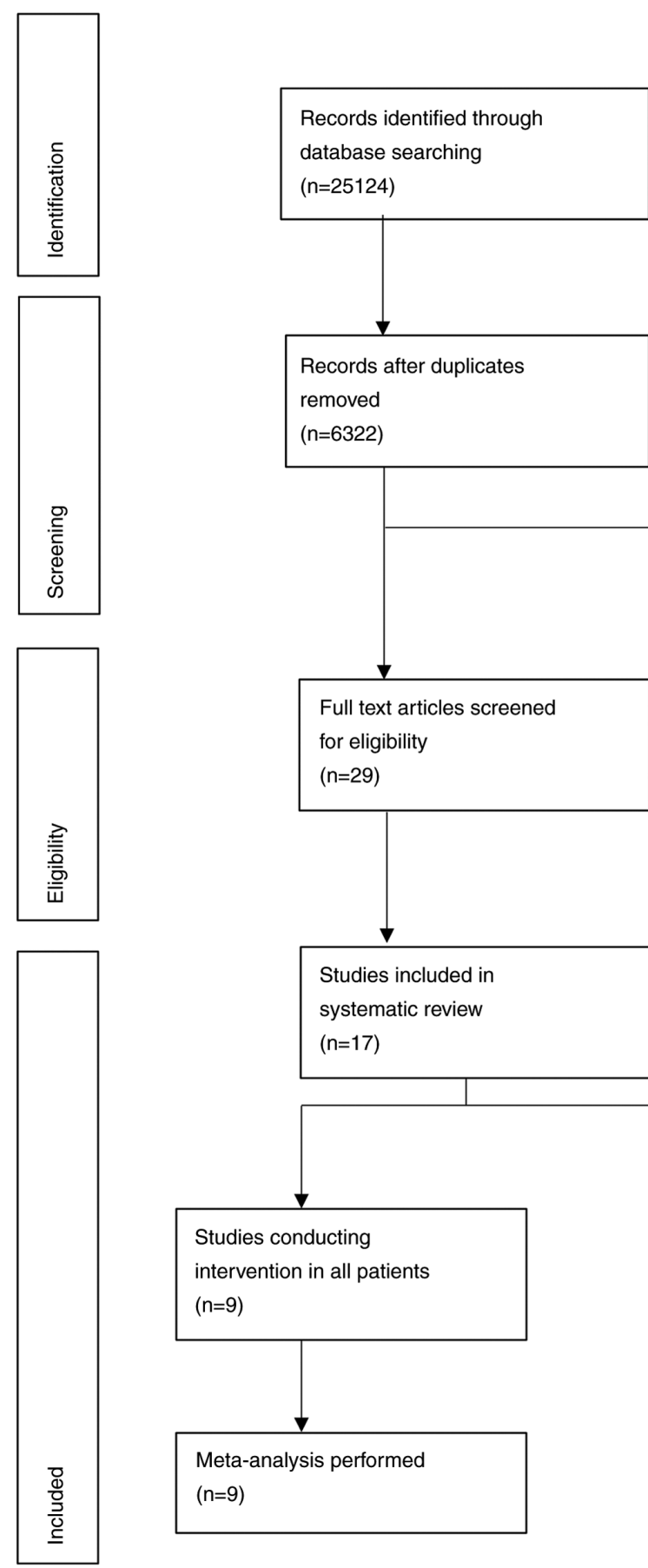

Records excluded after evaluation of

title/abstract due to non-relevance

$(n=6293)$
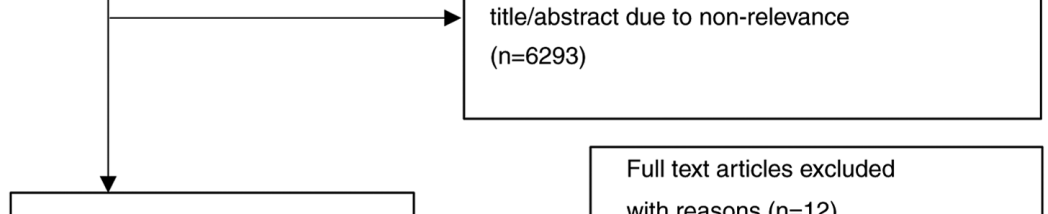

Full text articles screened

for eligibility

$(n=29)$

with reasons $(\mathrm{n}=12)$

-No control group $(n=2)$

-Non-English language $(n=2)$

-Not using mupirocin $(n=4)$

-No outcome data $(n=1)$

-Outcome data for cardiac

patients not available $(n=1)$

-Target population not

Studies included in

systematic review

$(n=17)$

specified $(n=1)$

-Groups not clearly defined

$(n=1)$
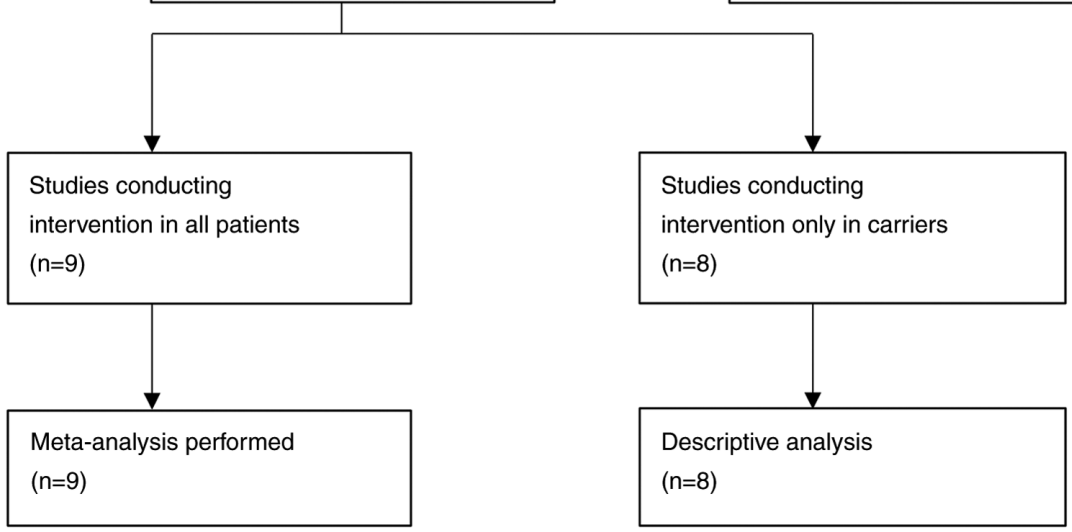

Figure 1. Study flow chart.

was used to calculate the pooled effect size for all analyses. The incidence of SSIs was compared between study and control groups using the Risk Ratio (RR) with 95\% CI. A sub-group analysis was also performed based on the use of other decolonization interventions along with mupirocin for the primary outcome. A leave-one-out analysis was performed to assess the influence of each study on the pooled effect size for both the primary and secondary outcome variables. Heterogeneity was assessed using the $\mathrm{I}^{2}$ statistic. $\mathrm{I}^{2}$ values of $25-50 \%$ represented low, values of $50-75 \%$ medium and $>75 \%$ represented substantial heterogeneity. Publication bias was not assessed due to the limited number of studies included in the meta-analysis $(<10)(20)$.

\section{Results}

Study retrieval and quality control. Initially, 6,322 unique records were identified in the literature search. Of these, 29 articles were selected for full-text review. A total of 12 studies were excluded as they did not fulfill the inclusion criteria (Fig. 1). A total of 17 studies were included (12-14,23-36). Since studies $(12,13,30-35)$ on targeted mupirocin-based decolonization were not coherent with respect to study groups and data presentation, only a descriptive analysis was performed. Meta-analysis was performed for studies (14,23-29,36) conducting universal decolonization with the mupirocin-based protocol. The results of the quality 


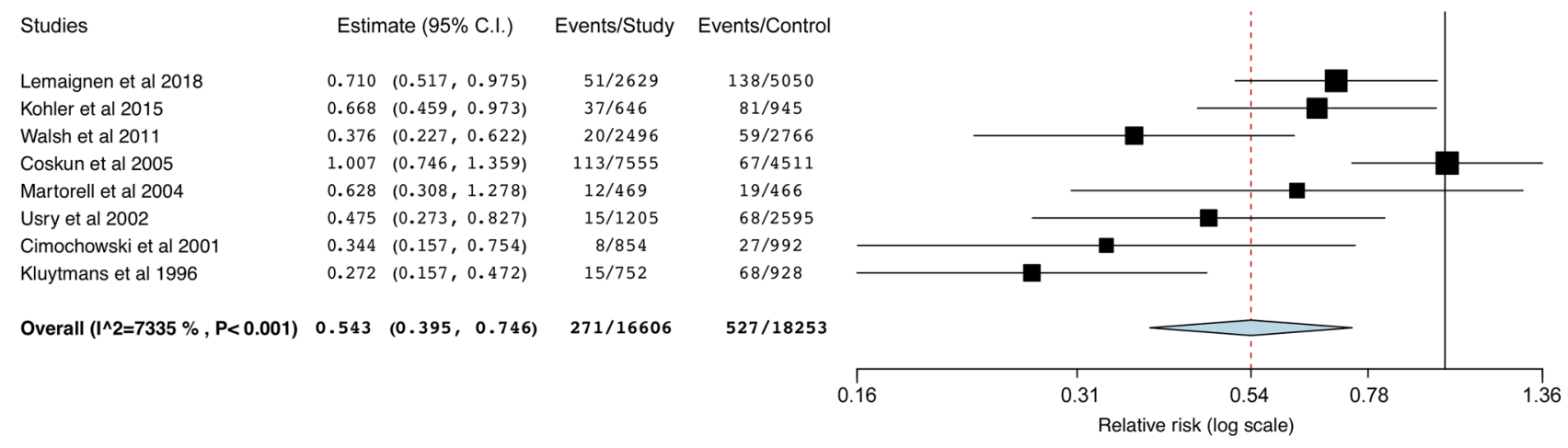

Figure 2. Forest plot of all surgical-site infections after universal mupirocin-based decolonization for all patients.

assessment of the included studies are presented in Table SII. The quality of RCTs was high but the overall quality of all remaining studies was low. Owing to their study designs, only two studies recruited patients in the same time period $(31,35)$. Multivariate analysis for confounding factors was performed only by three studies $(14,29,34)$.

Targeted mupirocin-based decolonization. A total of 8 studies $(12,13,30-35)$ assessed the role of targeted mupirocin-based decolonization on SSIs after a pre-operative screening of $S$. aureus carriers. The characteristics of these studies are presented in Table I. Of these studies, 4 were interrupted time-series (ITS) design studies (30,32-34), 1 was prospective (35), 1 was retrospective (31) and 2 were RCTs $(12,13)$. All studies were conducted in industrialized countries with 4 studies being carried out in the USA $(30,31,33,34)$. There were differences in the carrier states between the study and control groups in all 7 studies. Only carriers were included in both the study and control groups of the 2 RCTs $(12,13)$. Nicholas et al (35) and Shrestha et al (31) compared mupirocin-based decolonization between a study group of carriers and a control group of only non-carriers. In the remaining four studies, both carriers and non-carriers constituted the study and control groups (30,32-34), but the intervention was restricted to only carriers in the study group. S. aureus screening was carried out by either PCR or culture in the included studies. Decolonization protocol was restricted to the nasal application of mupirocin in three studies $(13,30,31)$ while the remaining five studies also utilized topical chlorhexidine (12,33-35) or triclosan (32) in the study groups. Duration of mupirocin use varied from 5-7 days. In four studies mupirocin was also used in the control groups pending results of carrier state screening (30-33). Three ITS studies also utilized additional antibiotic prophylaxis for carriers in the study group [vancomycin $(33,34)$ or teicoplanin $(32)]$.

In the four ITS studies (30,32-34), wherein screening and targeted mupirocin-based decolonization protocol was introduced, and data of pre-and post-intervention were compared; three reported a significant beneficial effect of the intervention $(30,32,34)$. Outcomes were, however, different in all three studies. Saraswat et al (34) reported significantly reduced incidence of all-SSIs, Jog et al (32) in MRSA-SSIs while Nicholson et al (30) only in SA-SSIs. Schweizer et al (33), in a multi-centric ITS study conducted on orthopedic and cardiac surgery patients, reported significantly reduced risk of SA-SSIs in the entire cohort [Incidence rate ratio (IRR): $0.58,95 \%$ CI: $0.37,0.92]$, but not specifically in patients undergoing cardiac surgery (IRR: 0.86, 95\% CI: 0.47,1.57). Amongst the 2 studies comparing carriers and non-carriers, the study by Nicholson and Huesman (30) did not report any data on SA-SSIs in the control group. They instead analyzed the efficacy of decolonization in the post-operative period and reported that successful decolonization significantly reduced the incidence of SA-SSIs. In another similar study, Shrestha et al (31) reported no difference in the incidence of SSIs between carriers and non-carriers and concluded that targeted decolonization in carriers leads to a significant reduction of mupirocin use without increasing SSIs in non-carriers. Finally, the RCT of Konvalinka et al (13) reported no statistically significant difference in SA-SSIs when mupirocin was used in a cohort of S. aureus carriers. However, in the RCT of Bode et al (12), a statistically significant reduction of SA-SSIs was noted with the use of nasal mupirocin and chlorhexidine soap.

Universal mupirocin-based decolonization. A total of 9 studies were included in the sub-group of studies using universal mupirocin-based decolonization (14,23-29,36). Details of the studies are presented in Table II. All studies were ITS design studies that introduced a universal mupirocin-based decolonization protocol without carrier state screening in their respective healthcare setups and analyzed its effect on the incidence of SSIs. A total of 4 studies $(23-25,36)$ used only nasal application of mupirocin in the decolonization protocol, while 4 studies $(14,26,27,29)$ additionally utilized anti-septic baths. In the study by Walsh et al (28), pre-operative screening of $S$. aureus carriers was performed and vancomycin was added to the pre-operative prophylaxis of carriers only. On the other hand, nasal mupirocin and coverage of chest tube and mediastinal tube exit site with mupirocin was used in all patients of the study group.

Except for the study by Thompson and Houston (27), all studies reported data on all SSIs in their respective cohorts. Analysis of the data of 34,859 patients revealed that the mupirocin-based universal decolonization protocol was associated with a significantly reduced risk of all SSIs (RR: 0.54; 95\% CI: $0.40,0.75 ; \mathrm{I}^{2}=73.35 \%$; Fig. 2). The "leave-one-out analysis' did not demonstrate any significant change in the outcome after the exclusion of one study at a time (Fig. 3). Sub-group 


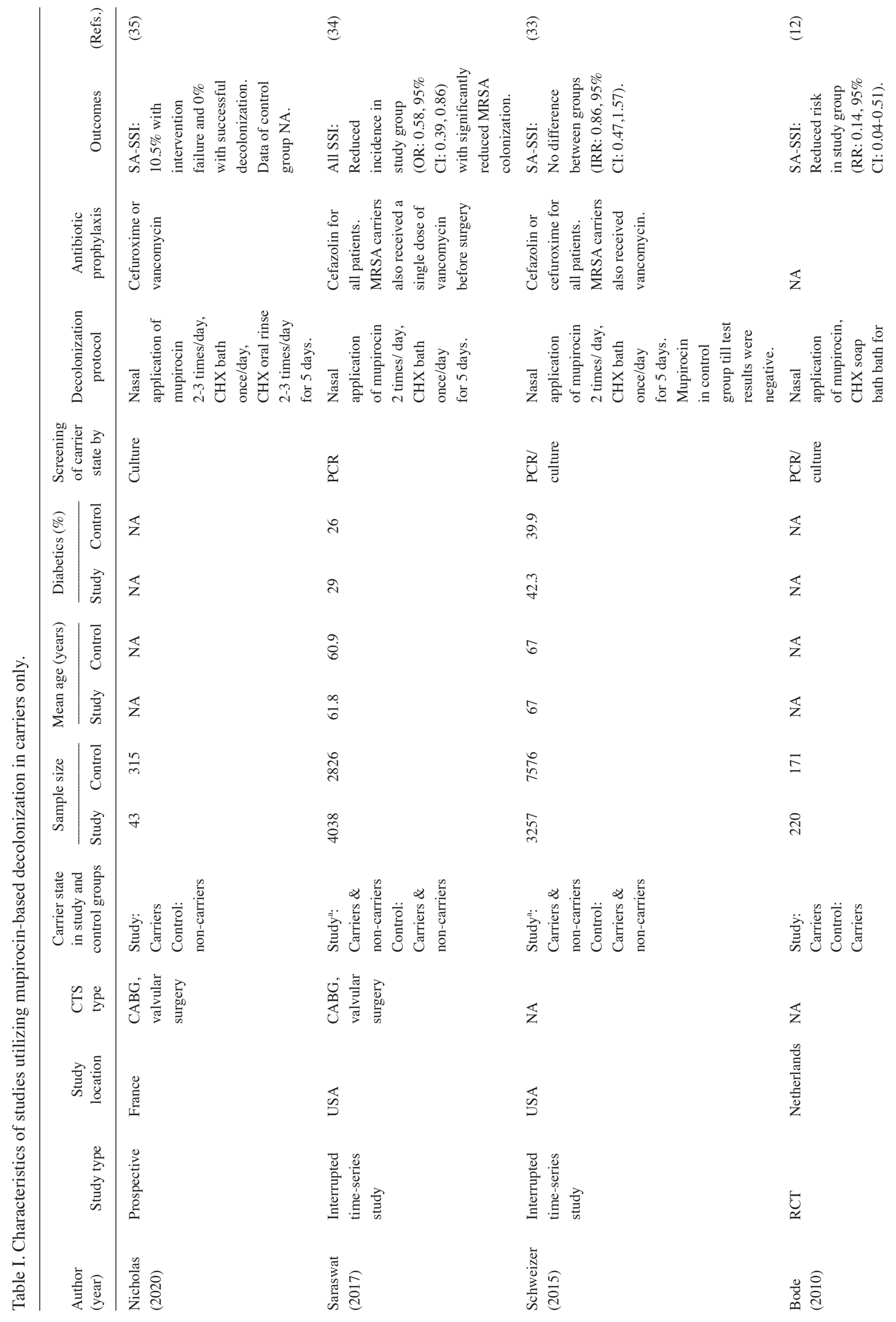




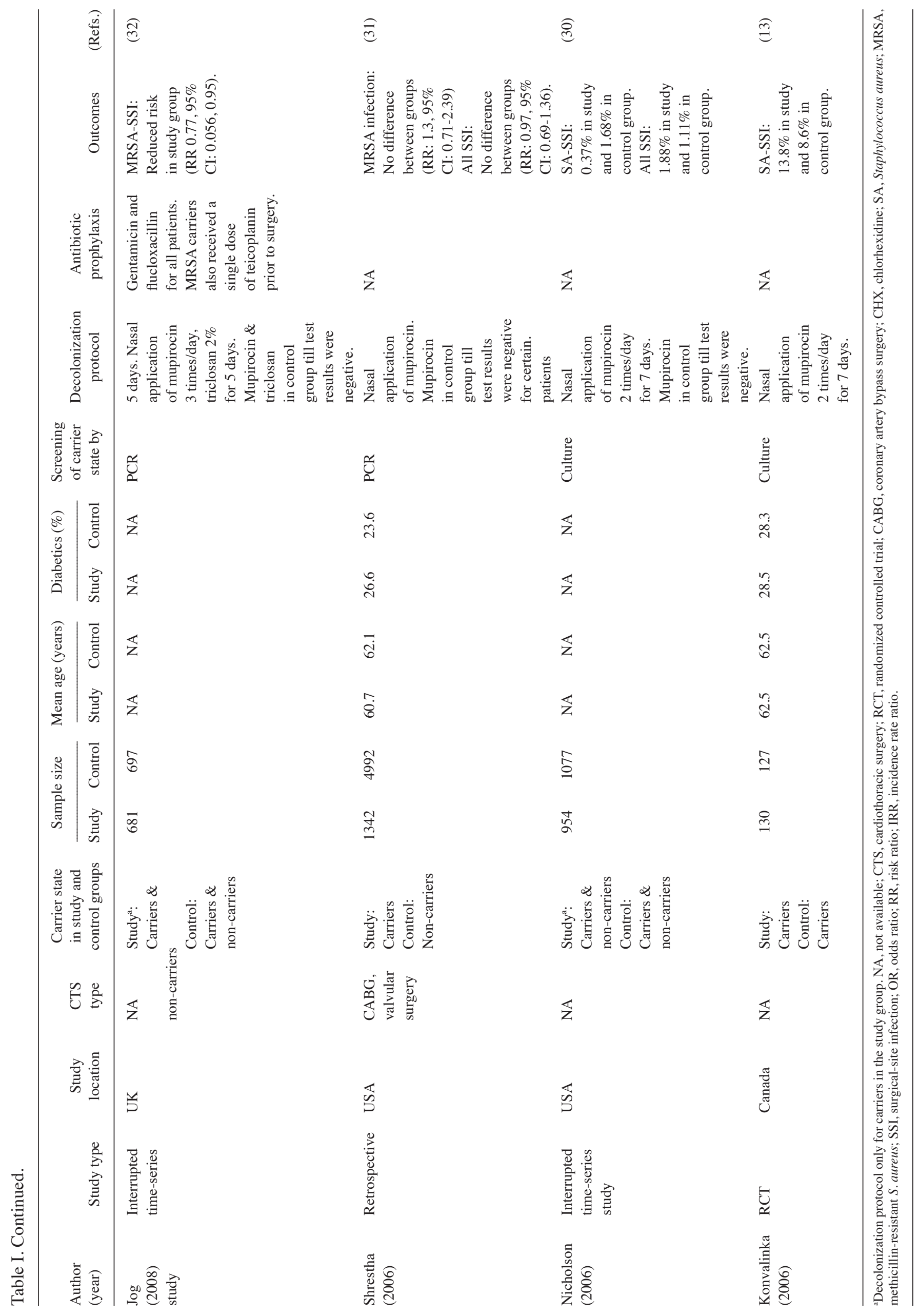




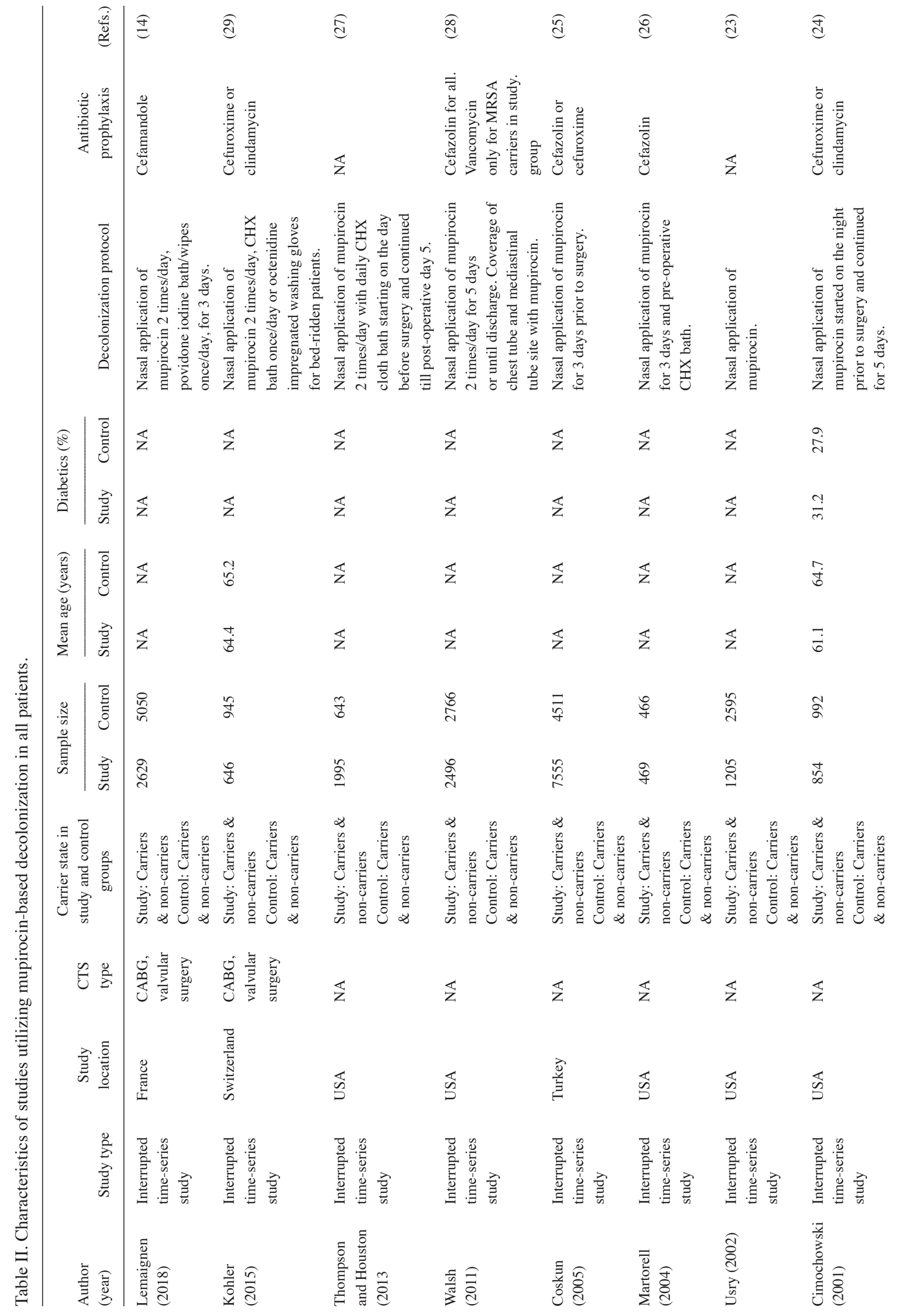


analysis based on use of other decolonization interventions with mupirocin (Yes vs. No) demonstrated reduction of all SSIs in both sub-groups (Yes-RR: 0.60; 95\% CI: 0.46,0.79; $I^{2}=35.39 \%$ and No-RR: 0.48 ; 95\% CI: $0.24,0.95 ; I^{2}=86.07 \%$; Fig. 4).

A total of 4 studies reported data on superficial and deep SSIs. Pooled analysis indicated a statistically significant reduction in the risk of superficial SSIs (RR: $0.3795 \%$ CI: 0.25,0.55; $\mathrm{I}^{2}=0 \%$; Fig. 5) in the study group, but no difference in deep SSIs between the two groups (RR: 0.45; 95\% CI: 0.19,1.09; $\mathrm{I}^{2}=80.67 \%$; Fig. 6). The leave-one-out analysis did not change the results of superficial SSIs, but on the exclusion of the study by Kohler et al (29), the results for deep SSIs were significantly in favor of mupirocin-based decolonization (RR: 0.31; 95\% CI: 0.18,0.52; data not shown). Data from 4 studies were pooled for SA-SSIs and MRSA-SSIs and the results indicated a significantly reduced risk of SA-SSIs in the study group (RR: $0.44 ; 95 \%$ CI: $0.32,0.61 ; I^{2}=0 \%$; Fig. 7 ) but not for MRSA-SSIs (RR: 0.25 ; 95\% CI: 0.05,1.28; $\mathrm{I}^{2}=79.07 \%$; Fig. 8). The results were stable in the leave-one-out analysis (data not shown).

\section{Discussion}

The present systematic review and meta-analysis of 17 studies separately analyzed evidence on the efficacy of targeted and universal mupirocin-based decolonization in preventing SSI in patients subjected to CT surgery. The role of targeted mupirocin-based decolonization remains inconclusive with contrasting evidence from RCTs and retrospective studies. Limited data from low-quality ITS studies suggested that universal mupirocin-based decolonization may be effective in reducing all SSIs, superficial SSIs and SA-SSIs with no effect on deep SSIs and MRSA-SSIs.

Since colonization with $S$. aureus has been known to increase the risk of nosocomial infections (7-9), decolonization protocols to reduce carriage and infection rates have been widely reported in the medical and surgical literature. According to a systematic review and meta-analysis by Gebreselassie et al (37), mupirocin with whole-body decolonization is highly effective in eradicating MRSA infestation in hemodialysis patients. Another study focusing on non-surgical patients reported a 59\% reduced risk of S. aureus infection in patients decolonized using nasal mupirocin (38). Orthopedic procedures including implant placement and joint replacement surgeries are associated with a high risk of developing SSI due to foreign body placement. Meta-analysis studies have demonstrated the effectiveness of nasal decolonization in reducing gram-positive SSIs and MRSA-SSIs in such patients $(17,18)$.

Mupirocin-based decolonization has not only been used to reduce transmission and infections in MRSA carriers but also to reduce all gram-positive SSIs $(25,26)$. Thus, controversy exists regarding whether such a decolonization strategy should be restricted to only high-risk carriers or whether a more universal decolonization protocol should be followed (39). To answer this question, Huang et al (40) conducted a large RCT involving 43 hospitals with 74,256 intensive care unit patients. They concluded that universal decolonization was more effective than screening and targeted decolonization to reduce bloodstream infections caused by any pathogen. 


\begin{tabular}{lccc} 
Studies & \multicolumn{2}{c}{ Estimate } & $(95 \%$ C.I. $)$ \\
Overall & 0.543 & $(0.395$, & $0.746)$ \\
& & & \\
- Lemaignen et al & 0.511 & $(0.347$, & $0.754)$ \\
- Kohler et al & 0.519 & $(0.355$, & $0.759)$ \\
- Walsh et al & 0.574 & $(0.412$, & $0.800)$ \\
- Coskun et al & 0.491 & $(0.368$, & $0.656)$ \\
- Martorell et al & 0.532 & $(0.375$, & $0.754)$ \\
- Usry et al & 0.550 & $(0.387$, & $0.782)$ \\
- Cimochowski et al & 0.567 & $(0.407$, & $0.789)$ \\
- Kluytmans et al & 0.607 & $(0.455$, & $0.808)$
\end{tabular}

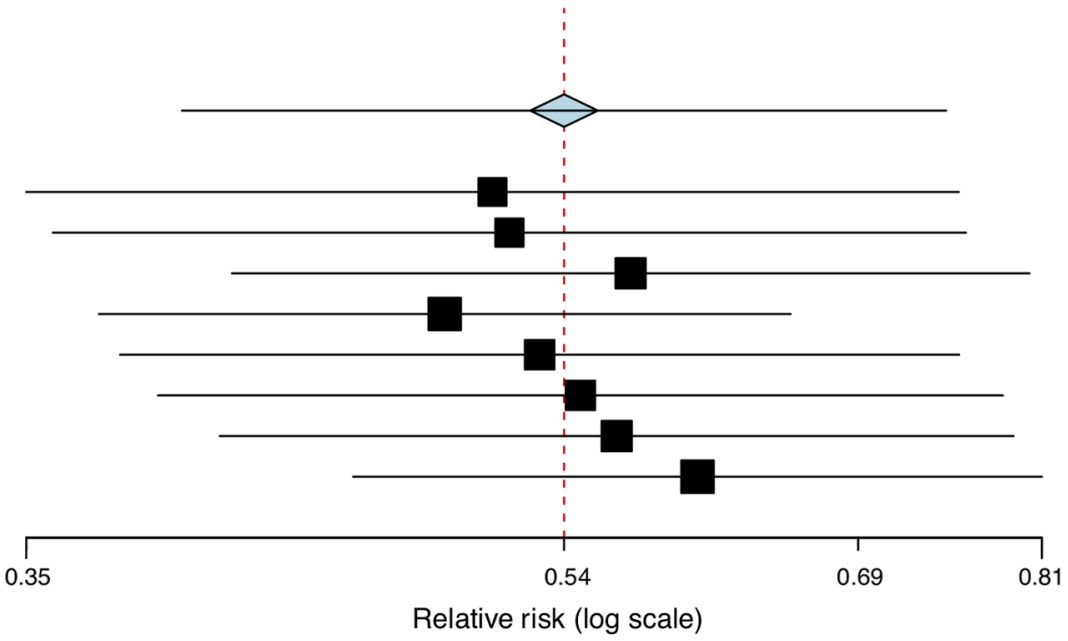

Figure 3. Leave-one-out analysis of all surgical-site infections for studies utilizing universal mupirocin-based decolonization.

Studies
Lemaignen et al
Kohler et al
Walsh et al
Martorell et al
Subgroup Yes (I^2=3539\%, P=0.200)
Coskun et al
Usry et al
Cimochowski et al
Kluytmans et al
Subgroup No $\left(\mathbf{I}^{\wedge} \mathbf{2}=\mathbf{8 6 0 7 \%}, \mathbf{P}=\mathbf{0 . 0 0 0}\right)$

Overall (I^2=7335\%, P=0.000)

$$
\text { Estimate (95\% C.I.) }
$$

$0.710(0.517,0.975)$

$0.668(0.459,0.973)$

$0.376(0.227,0.622)$

$0.628(0.308,1.278)$

$0.601(0.457,0.791)$

$1.007 \quad(0.746,1.359)$

$0.475(0.273,0.827)$

$0.344(0.157,0.754)$

$0.272(0.157,0.472)$

$0.477(0.239,0.950)$

$0.543(0.395,0.746)$
Events/Study Events/Control

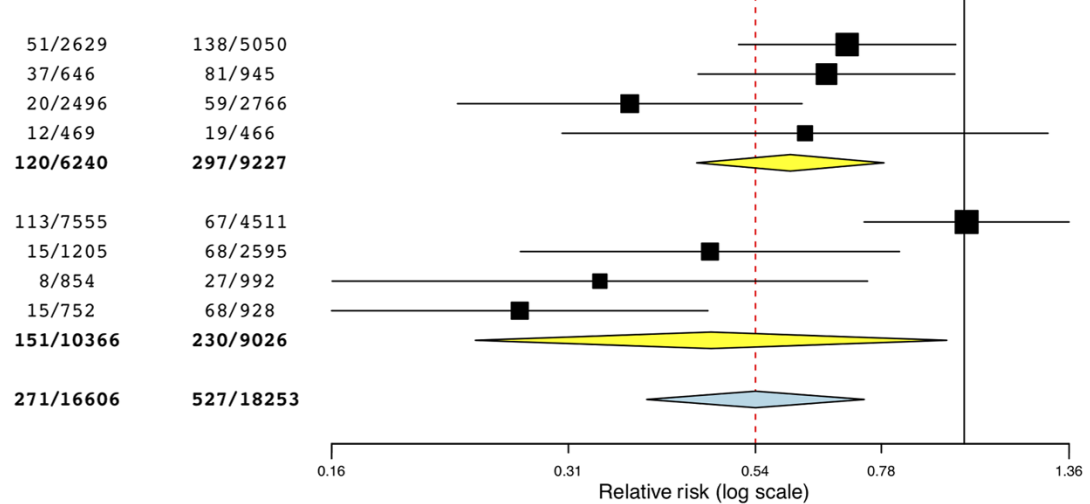

Figure 4. Sub-group analysis based on the use of other decolonization interventions (Yes vs. No) with mupirocin for all surgical-site infections.

Studies

Kohler et al 2015

Usry et al 2002

Cimochowski et al 2001

Kluytmans et al 1996

Overall $\left(\left.\right|^{\wedge} 2=0 \%, P=0.831\right) \quad 0.369 \quad(0.248,0.549)$
Events/Study Events/Control

$\begin{array}{cl}10 / 646 & 48 / 945 \\ 6 / 1205 & 25 / 2595 \\ 5 / 854 & 15 / 992 \\ 9 / 752 & 31 / 928\end{array}$

$30 / 3457 \quad 119 / 5460$

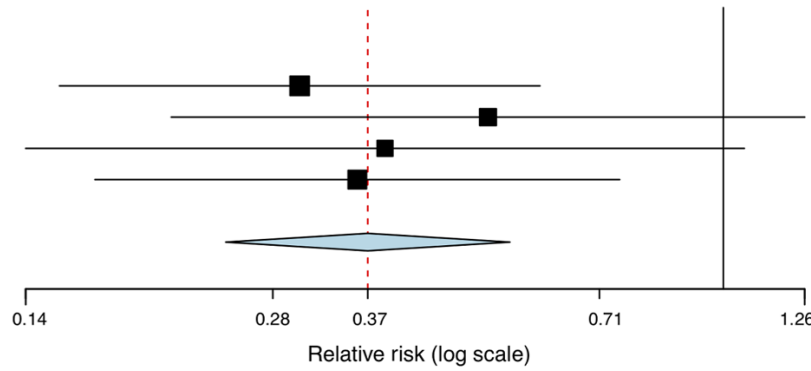

Figure 5. Forest plot of superficial surgical-site infections after universal mupirocin-based decolonization.

To provide comprehensive and clear evidence on the efficacy of both protocols in patients subjected to CT, studies assessing targeted and universal mupirocin-based decolonization were separated for the present review. Descriptive analysis of targeted decolonization in CT surgical patients indicated contrasting results among the included studies. Amongst the RCTs, Bode et al (12) reported a significantly reduced risk of SA-SSIs but Konvalinka et al (13) indicated no significant difference in their study sample. The slightly larger sample size and use of chlorhexidine soap may have influenced outcomes in the trial performed by Bode et al (12).
Similarly, for ITS-design studies on mupirocin-based targeted decolonization, owing to differences in study outcomes and varying results, it was not possible to draw any strong conclusions. Saraswat et al (34) reported a reduced risk of all-SSIs in their cohort but no such effect was noted by Nicholson and Huesman (30). Similarly, results were not coherent for the incidence of SA-SSIs and only Jog et al (32) reported data on MRSA-SSIs. Differences in the study population, decolonization protocol and other infection control measures in the included studies may have contributed to these differences. 


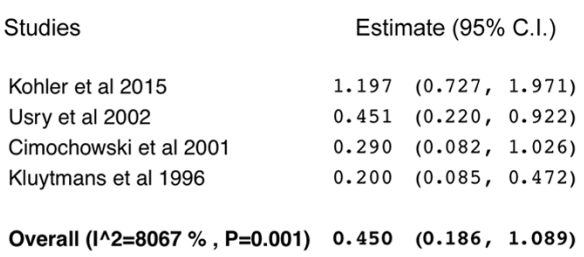

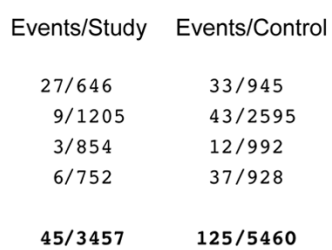

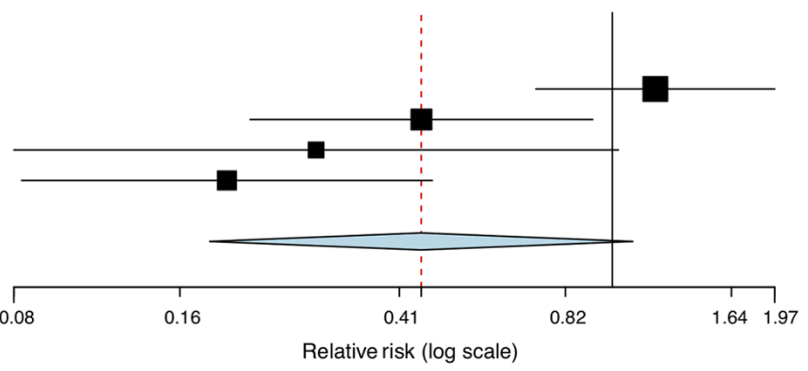

Figure 6. Forest plot of deep surgical-site infections after universal mupirocin-based decolonization.

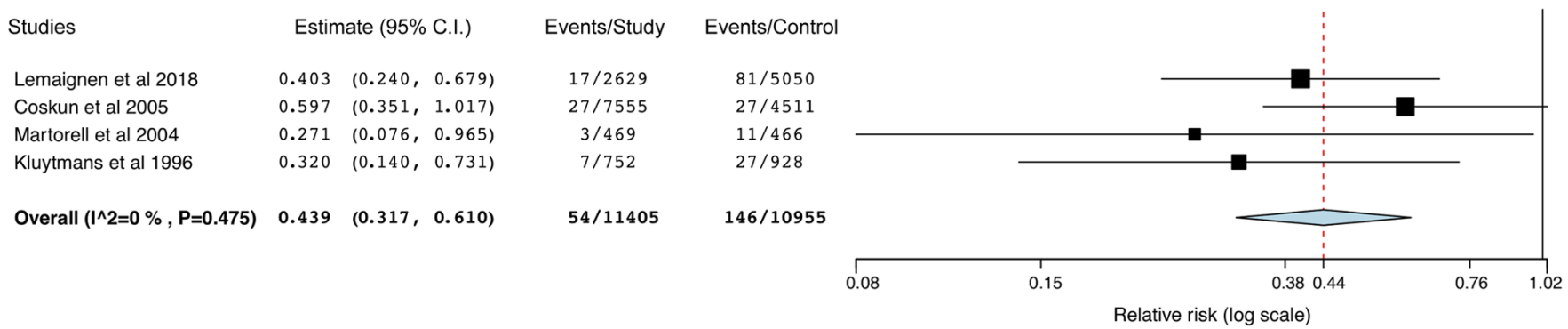

Figure 7. Forest plot of S. aureus surgical-site infections after universal mupirocin-based decolonization.

\begin{tabular}{lrrr} 
Studies & \multicolumn{3}{c}{ Estimate $(95 \%$ C.I. $)$} \\
Thompson et al 2013 & $0.040(0.005,0.322)$ \\
Walsh et al 2011 & $0.069(0.017,0.289)$ \\
Coskun et al 2005 & 0.682 & $(0.333,1.397)$ \\
Martorell et al 2004 & $1.987(0.181,21.840)$ \\
Overall (I^2=7907\%, P=0.002) & $\mathbf{0 . 2 4 5}$ & $(\mathbf{0 . 0 4 7}, 1.277)$
\end{tabular}
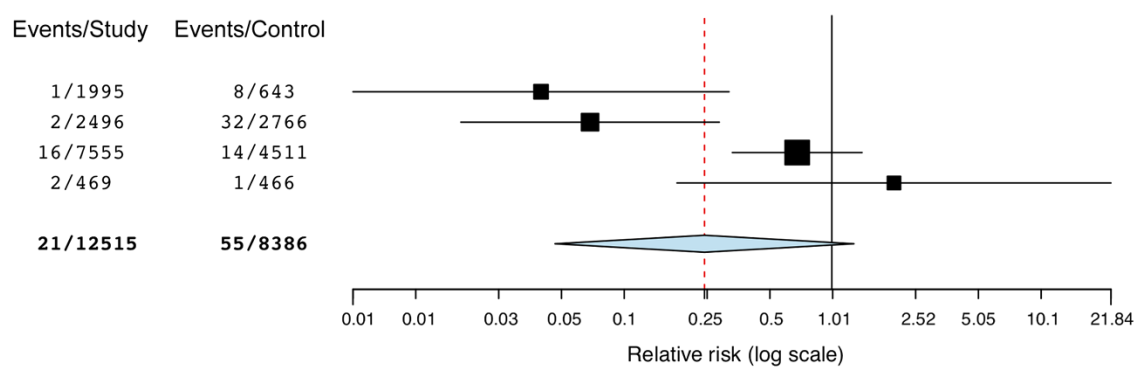

Figure 8. Forest plot of methicillin-resistant S. aureus surgical-site infections after universal mupirocin-based decolonization.

Targeted decolonization has been recommended to reduce the emergence of mupirocin resistance (41). The literature on the level of mupirocin resistance following its universal use is, however, scarce. The REDUCE-MRSA trial (42) compared targeted and universal decolonization with mupirocin and chlorhexidine and determined that the odds of mupirocin resistance were not different across treatment arms. The results were not conclusive, as the $95 \%$ CIs of the outcomes were wide. In a recent meta-analysis, Dadashi et al (41) reported an increased prevalence of mupirocin resistance in both $S$. aureus and MRSA, indicating that reduced efficacy of mupirocin poses a risk for invasive infection. In one of the included studies, Shrestha et al (31) demonstrated that limiting mupirocin to only carriers does not increase the risk of MRSA and all-SSIs in non-carriers. In recent years, due to the increased awareness of mupirocin resistance, research is underway to identify alternative nasal decolonization agents. Alcohol and iodine-based agents are in use but their efficacy is still under investigation (15).

Despite the drawback of anti-microbial resistance, universal decolonization with mupirocin offers certain distinct advantages. MRSA surveillance and the requirement for contact precautions may be eliminated with universal decolonization, which may reduce hospital costs and contribute to better patient care $(40,43)$. It may also reduce the total microbial burden of the patient and contribute to a greater reduction in hospital infection rates (40). The present analysis of 9 studies revealed that mupirocin-based decolonization was effective in reducing the risk of all SSIs, superficial SSIs and SA-SSIs in CT surgical patients but not in reducing deep SSIs and MRSA-SSIs. The lack of significant results for MRSA-SSIs may be due to the limited number of studies reporting data on MRSA-SSIs. In addition, the exact number of MRSA carriers in the study and control groups was unknown. An insufficient number of carriers in these studies may have led to reduced statistical power.

The results of the present analysis concur with previous reviews. Schweizer et al (17), in a review of studies published up to January 2012, reported a significantly reduced risk of gram-positive SSIs and SA-SSIs with nasal decolonization in cardiac surgery patients but no difference in MRSA infection rates. In 2017, Ma et al (18), in a meta-analysis of 9 studies on pediatric and adult cardiac surgical patients, determined a significantly reduced risk of all SSIs and SA-SSIs with 
nasal decolonization. In contrast to the present analysis, these reviews were not focussed on mupirocin-based decolonization and included a mix of studies employing both targeted and universal decolonization.

The decolonization protocol was not identical among the included studies. Anti-septic baths consisting of chlorhexidine and povidone-iodine were used with mupirocin in half of the included studies. It is known that $S$. aureus may colonize other regions of the body, including the pharynx, groin, perianal region or axilla and contribute to SSIs (12). Therefore, it is plausible that the use of chlorhexidine for whole-body decolonization with mupirocin for nostrils may lead to a greater reduction of SSIs (44). However, the current evidence on the role of such anti-septic baths for reducing SSIs is weak. Meta-analysis studies have reported no significant reduction in the risk of SSIs with anti-septic baths $(45,46)$. A total of 4 studies included in the present review also used additional glycopeptide antibiotics for MRSA carriers along with mupirocin-based decolonization. Saleh et al (47) demonstrated that the addition of glycopeptide antibiotics significantly reduces the risk of $S$. aureus infections in patients undergoing cardiac, vascular and orthopedic surgery. To analyze the role of such additional interventions on the pooled outcomes of the present meta-analysis, sub-group and leave-one-out analyses were performed. The exclusion of the single study by Walsh et al (28), in which vancomycin was added to MRSA carriers in the universal decolonization study, did not change the significance of the overall results. In addition, on subgroup analysis, no difference in the risk of all SSIs was identified for studies using these additional interventions.

The limitations of the present review require to be mentioned. First, due to the limited number of coherent studies, it was not possible to perform any meta-analysis for targeted mupirocin-based decolonization. Furthermore, the results of the pooled analysis of data from retrospective ITS studies may be limited due to the high risk of bias. Time-based variation in the hospital infection control protocol, as well as differences in surgical technique, compliance of intervention or level of post-operative care may have skewed the results. Geographical variation in the patient population with baseline differences in patient comorbidities and the type of surgical procedure may also have influenced infection rates. In addition, the decolonization protocol was not similar across studies with the use of antiseptic baths and glycopeptide antibiotic prophylaxis in certain studies. Finally, only four studies were included in the meta-analysis of secondary outcomes, which limited the power of the analysis.

However, the present study provides up-to-date and comprehensive evidence on the role of mupirocin-based decolonization in CT surgical patients. Studies were segregated based on the decolonized population, analyzing them separately for available evidence. Appropriate sub-group and sensitivity analyses were performed to take into account differences among the included studies.

To conclude, the present review indicated that currently available evidence on the role of targeted mupirocin-based decolonization to reduce SSIs after CT surgery is non-coherent and inconclusive. Analysis of low-quality retrospective studies suggested that universal mupirocin-based decolonization may reduce all SSIs, superficial SSIs and SA-SSIs but not deep SSIs and MRSA-SSIs in CT surgical patients. Further, high-quality RCTs with a homogenous decolonization protocol on patients subjected to CT surgery are required to strengthen the current evidence.

\section{Acknowledgements}

Not applicable.

\section{Funding}

No funding received.

\section{Availability of data and materials}

The datasets used and/or analyzed during the current study are available from the corresponding author on reasonable request.

\section{Authors' contributions}

LW conceived and designed the study. QJ and XH collected the data and performed the literature search. LW was involved in the writing of the manuscript. All authors have read and approved the final manuscript.

\section{Ethics approval and consent to participate}

Not applicable.

\section{Patient consent for publication}

Not applicable.

\section{Competing interests}

The authors declare that they have no competing interests.

\section{References}

1. Loop FD, Lytle BW, Cosgrove DM, Mahfood S, McHenry MC, Goormastic M, Stewart RW, Golding LA and Taylor PC: J Maxwell Chamberlain memorial paper. Sternal wound complications after isolated coronary artery bypass grafting: Early and late mortality, morbidity, and cost of care. Ann Thorac Surg 49: 179-186; discussion 186-7, 1990.

2. Lepelletier D, Perron S, Bizouarn P, Caillon J, Drugeon H, Michaud JL and Duveau D: Surgical-site infection after cardiac surgery: Incidence, microbiology, and risk factors. Infect Control Hosp Epidemiol 26: 466-472, 2005.

3. Saadatian-Elahi M, Teyssou R and Vanhems P: Staphylococcus aureus, the major pathogen in orthopaedic and cardiac surgical site infections: A literature review. Int J Surg 6: 238-245, 2008.

4. Abboud CS, Wey SB and Baltar VT: Risk factors for mediastinitis after cardiac surgery. Ann Thorac Surg 77: 676-683, 2004.

5. Grossi EA, Culliford AT, Krieger KH, Kloth D, Press R, Baumann FG and Spencer FC: A survey of 77 major infectious complications of median sternotomy: A review of 7,949 consecutive operative procedures. Ann Thorac Surg 40: 214-223, 1985.

6. Filsoufi F, Castillo JG, Rahmanian PB, Broumand SR, Silvay G, Carpentier A and Adams DH: Epidemiology of deep sternal wound infection in cardiac surgery. J Cardiothorac Vasc Anesth 23: 488-494, 2009.

7. Verhoeven PO, Gagnaire J, Botelho-Nevers E, Grattard F, Carricajo A, Lucht F, Pozzetto B and Berthelot P: Detection and clinical relevance of Staphylococcus aureus nasal carriage: An update. Expert Rev Anti Infect Ther 12: 75-89, 2014 
8. Gagnaire J, Verhoeven PO, Grattard F, Rigaill J, Lucht F, Pozzetto B, Berthelot $P$ and Botelho-Nevers E: Epidemiology and clinical relevance of Staphylococcus aureus intestinal carriage: A systematic review and meta-analysis. Expert Rev Anti Infect Ther 15: 767-785, 2017.

9. Katayanagi T: Nasal methicillin-resistant $S$. aureus is a major risk for mediastinitis in pediatric cardiac surgery. Ann Thorac Cardiovasc Surg 21: 37-44, 2015

10. van Rijen M, Bonten M, Wenzel R and Kluytmans J: Mupirocin ointment for preventing Staphylococcus aureus infections in nasal carriers. Cochrane Database Syst Rev 4: CD006216, 2008.

11. Langenberg JCM, Kluytmans JAJW, Mulder PGH, Romme J, Ho GH and Van Der Laan L: Peri-Operative nasal eradication therapy prevents staphylococcus aureus surgical site infections in aortoiliac surgery. Surg Infect (Larchmt) 19: 510-515, 2018.

12. Bode LG, Kluytmans JA, Wertheim HF, Bogaers D Vandenbroucke-Grauls CM, Roosendaal R, Troelstra A, Box AT, Voss A, van der Tweel I, et al: Preventing surgical-site infections in nasal carriers of Staphylococcus aureus. N Engl J Med 362: 9-17, 2010

13. Konvalinka A, Errett L and Fong IW: Impact of treating Staphylococcus aureus nasal carriers on wound infections in cardiac surgery. J Hosp Infect 64: 162-168, 2006.

14. Lemaignen A, Armand-Lefevre L, Birgand G, Mabileau G, Lolom I, Ghodbane W, Dilly MP, Nataf P and Lucet JC: Thirteen-year experience with universal Staphylococcus aureus nasal decolonization prior to cardiac surgery: A quasi-experimental study. J Hosp Infect 100: 322-328, 2018.

15. Sakr A, Brégeon F, Rolain JM and Blin O: Staphylococcus aureus nasal decolonization strategies: A review. Expert Rev Anti Infect Ther 17: 327-340, 2019.

16. Liu Z, Norman G, Iheozor-Ejiofor Z, Wong JK, Crosbie EJ and Wilson P: Nasal decontamination for the prevention of surgical site infection in Staphylococcus aureus carriers. Cochrane Database Syst Rev 5: CD012462, 2017.

17. Schweizer M, Perencevich E, McDanel J, Carson J, Formanek M, Hafner J, Braun B and Herwaldt L: Effectiveness of a bundled intervention of decolonization and prophylaxis to decrease Gram positive surgical site infections after cardiac or orthopedic surgery: Systematic review and meta-analysis. BMJ 346: f2743, 2013.

18. Ma N, Cameron A, Tivey D, Grae N, Roberts S and Morris A: Systematic review of a patient care bundle in reducing staphylococcal infections in cardiac and orthopaedic surgery. ANZ J Surg 87: 239-246, 2017.

19. Moher D, Liberati A, Tetzlaff J, Altman DG and PRISMA Group: Preferred reporting items for systematic reviews and meta-analyses: The PRISMA statement. BMJ 339: b2535, 2009.

20. Higgins JPT, Thomas J, Chandler J, Cumpston M, Li T, Page MJ and Welch VA: Cochrane Handbook for Systematic Reviews of Interventions. Version 6.1 (updated September 2020). Cochrane, 2020. Available from www.training.cochrane.org/handbook

21. Kim SY, Park JE, Lee YJ, Seo HJ, Sheen SS, Hahn S, Jang BH and Son HJ: Testing a tool for assessing the risk of bias for nonrandomized studies showed moderate reliability and promising validity. J Clin Epidemiol 66: 408-414, 2013.

22. Wallace BC, Schmid CH, Lau J and Trikalinos TA: Meta-analyst: Software for meta-analysis of binary, continuous and diagnostic data. BMC Med Res Methodol 9: 80, 2009.

23. Usry GH, Johnson L, Weems JJ Jr and Blackhurst D: Process improvement plan for the reduction of sternal surgical site infections among patients undergoing coronary artery bypass graft surgery. Am J Infect Control 30: 434-436, 2002.

24. Cimochowski GE, Harostock MD, Brown R, Bernardi M, Alonzo N and Coyle K: Intranasal mupirocin reduces sternal wound infection after open heart surgery in diabetics and nondiabetics. Ann Thorac Surg 71: 1572-1578; discussion 1578-9, 2001.

25. Coskun D and Aytac J: Decrease in Staphylococcus aureus surgical site infections following cardiovascular surgery. J Hosp Infect 60: 287-289, 2005

26. Martorell C, Engelman R, Corl A and Brown RB: Surgical site infections in cardiac surgery: An 11-year perspective. Am J Infect Control 32: 63-68, 2004.

27. Thompson P and Houston S: Decreasing methicillin-resistant Staphylococcus aureus surgical site infections with chlorhexidine and mupirocin. Am J Infect Control 41: 629-633, 2013.

28. Walsh EE, Greene L and Kirshner R: Sustained reduction in methicillin-resistant Staphylococcus aureus wound infections after cardiothoracic surgery. Arch Intern Med 171: 68-73, 2011.

29. Kohler P, Sommerstein R, Schönrath F, Ajdler-Schäffler E, Anagnostopoulos A, Tschirky S, Falk V, Kuster SP and Sax H: Effect of perioperative mupirocin and antiseptic body wash on infection rate and causative pathogens in patients undergoing cardiac surgery. Am J Infect Control 43: e33-e38, 2015.
30. Nicholson MR and Huesman LA: Controlling the usage of intranasal mupirocin does impact the rate of Staphylococcus aureus deep sternal wound infections in cardiac surgery patients. Am J Infect Control 34: 44-48, 2006.

31. Shrestha NK, Banbury MK, Weber M, Cwynar RE, Lober C, Procop GW, Karafa MT and Gordon SM: Safety of targeted perioperative mupirocin treatment for preventing infections after cardiac surgery. Ann Thorac Surg 81: 2183-2188, 2006.

32. Jog S, Cunningham R, Cooper S, Wallis M, Marchbank A, Vasco-Knight P and Jenks PJ: Impact of preoperative screening for meticillin-resistant Staphylococcus aureus by real-time polymerase chain reaction in patients undergoing cardiac surgery. J Hosp Infect 69: 124-130, 2008.

33. Schweizer ML, Chiang HY, Septimus E, Moody J, Braun B, Hafner J, Ward MA, Hickok J, Perencevich EN, Diekema DJ, et al: Association of a bundled intervention with surgical site infections among patients undergoing cardiac, hip, or knee surgery. JAMA 313: 2162-2171, 2015.

34. Saraswat MK, Magruder JT, Crawford TC, Gardner JM, Duquaine D, Sussman MS, Maragakis LL and Whitman GJ: Preoperative staphylococcus aureus screening and targeted decolonization in cardiac surgery. Ann Thorac Surg 104: 1349-1356, 2017

35. Nicolas R, Carricajo A, Morel J, Rigaill J, Grattard F, Guezzou S, Audoux E, Campisi S, Favre JP, Berthelot P, et al: Evaluation of effectiveness and compliance with the mupirocin nasal ointment part of Staphylococcus aureus decolonization in real life using UPLC-MS/MS mupirocin quantification. J Antimicrob Chemother 75: 1623-1630, 2020.

36. Kluytmans JA, Mouton JW, VandenBergh MF, Manders MJ, Maat AP, Wagenvoort JH, Michel MF and Verbrugh HA: Reduction of surgical-site infections in cardiothoracic surgery by elimination of nasal carriage of staphylococcus aureus. Infect Control Hosp Epidemiol 17: 780-785, 1996.

37. Gebreselassie HM, Lo Priore E and Marschall J: Effectiveness of meticillin-resistant Staphylococcus aureus decolonization in long-term haemodialysis patients: A systematic review and meta-analysis. J Hosp Infect 91: 250-256, 2015.

38. Nair R, Perencevich EN, Blevins AE, Goto M, Nelson RE and Schweizer ML: Clinical effectiveness of mupirocin for preventing staphylococcus aureus infections in nonsurgical settings: A meta-analysis. Clin Infect Dis 62: 618-630, 2016.

39. Wenzel RP and Edmond MB: Infection control: The case for horizontal rather than vertical interventional programs. Int J Infect Dis 14 (Suppl 4): S3-S5, 2010.

40. Huang SS, Septimus E, Kleinman K, Moody J,Hickok J, Avery TR, Lankiewicz J, Gombosev A, Terpstra L, Hartford F, et al: Targeted versus universal decolonization to prevent ICU infection. N Engl J Med 368: 2255-2265, 2013.

41. Dadashi M, Hajikhani B, Darban-Sarokhalil D, van Belkum A and Goudarzi M: Mupirocin resistance in Staphylococcus aureus: A systematic review and meta-analysis. J Glob Antimicrob Resist 20: 238-247, 2020.

42. Hayden MK, Lolans K, Haffenreffer K, Avery TR, Kleinman K, Li H, Kaganov RE, Lankiewicz J, Moody J, Septimus E, et al: Chlorhexidine and mupirocin susceptibility of methicillinresistant staphylococcus aureus isolates in the REDUCE-MRSA trial. J Clin Microbiol 54: 2735-2742, 2016.

43. Morgan DJ, Diekema DJ, Sepkowitz K and Perencevich EN: Adverse outcomes associated with contact precautions: A review of the literature. Am J Infect Control 37: 85-93, 2009.

44. McConeghy KW, Mikolich DJ and LaPlante KL: Agents for the decolonization of methicillin-resistant Staphylococcus aureus. Pharmacotherapy 29: 263-280, 2009.

45. Webster J and Osborne S: Preoperative bathing or showering with skin antiseptics to prevent surgical site infection. Cochrane Database Syst Rev CD004985, 2015.

46. Franco LM, Cota GF, Pinto TS and Ercole FF: Preoperative bathing of the surgical site with chlorhexidine for infection prevention: Systematic review with meta-analysis. Am J Infect Control 45: 343-349, 2017.

47. Saleh A, Khanna A, Chagin KM, Klika AK, Johnston D and Barsoum WK: Glycopeptides versus $\beta$-lactams for the prevention of surgical site infections in cardiovascular and orthopedic surgery: A mata-analysis. Ann Surg 261: 72-80, 2015.

This work is licensed under a Creative Common Attribution-NonCommercial-NoDerivatives 4.0 International (CC BY-NC-ND 4.0) License. 